\title{
Should intramuscular clozapine be adopted into mainstream clinical practice?
}

\author{
Shaun Bhattacherjee, Anthony Young, Niraj Ahuja and Steven Allen
}

\begin{abstract}
Summary
Clozapine is under-used in the UK, and Casetta et al's recent paper in the BJPsych adds to a growing number of small studies that support the use of intramuscular clozapine to initiate and maintain treatment with oral clozapine. However, intramuscular clozapine remains unlicensed and, because of the risks associated with its administration, it should be used only cautiously before it can be adopted more widely into mainstream clinical practice.
\end{abstract}

\section{Keywords}

Antipsychotics; schizophrenia; in-patient treatment; outcome studies; psychotic disorders.

\section{Copyright and usage}

(c) The Author(s), 2021. Published by Cambridge University Press on behalf of the Royal College of Psychiatrists.
Shaun Bhattacherjee currently works as a consultant psychiatrist and Assistant Medical Director at the Cumbria, Northumberland, Tyne and Wear NHS Foundation Trust, UK. Anthony Young is Acting Deputy Chief Pharmacist at the Cumbria,

Northumberland, Tyne and Wear NHS Foundation Trust and an honorary lecturer at the Sunderland University School of Pharmacy, UK. Niraj Ahuja is a consultant psychiatrist and Assistant Medical Director at the Cumbria, Northumberland, Tyne and Wear NHS Foundation Trust and an associate clinical lecturer at Newcastle University, UK. Steven Allen is a senior clinical pharmacist at the Cumbria, Northumberland, Tyne and Wear NHS Foundation Trust, working on the acute adult and older people's mental health wards in Newcastle upon Tyne, UK.

Antipsychotic medication has played an important role in psychiatric care and contributed significantly to an eightfold reduction in in-patient bed use in the nearly 70 years since chlorpromazine was first described in $1952 .{ }^{1}$ However, approximately $30 \%$ of people with schizophrenia are treatment resistant and, of these, $50-75 \%$ respond to treatment with clozapine. Clozapine is associated with improved long-term outcomes; most significantly, a reduced mortality from both natural and unnatural causes, a reduction in violent offending and reduced readmission rates. ${ }^{2}$

Clozapine is, however, under-used, partly because of concerns about the risk of immediate or long-term non-concordance with oral medication and partly because of concerns regarding its sideeffects profile, its safety when initiated in community settings, the inconvenience of regular blood testing, the need to carefully titrate its dose and the need to re-titrate after any short gap in treatment. ${ }^{3}$

Intramuscular clozapine has been used in high secure and other forensic settings for many years. Consequently, intramuscular administration can be seen as a potentially useful short- or even medium-term strategy to facilitate the initiation of oral clozapine as well as to prevent gaps in treatment (thus potentially reducing the risk of relapse and the need to re-titrate for patients who become temporarily non-concordant with oral medication).

\section{Casetta et al's study}

There are several small studies, from both the UK and elsewhere, describing this use of intramuscular clozapine. In a recent issue of the BJPsych, Casetta and colleagues ${ }^{2}$ further make a case for more widespread use of this drug by sharing their experience of intramuscular clozapine use in detained patients with treatment-resistant psychosis over 3 years in the South London and Maudsley NHS Trust. Their retrospective study, using an anonymised case register, found that $92 \%$ of patients prescribed intramuscular clozapine subsequently became concordant with oral clozapine; this seems impressive and may have significant implications for mainstream clinical practice.

However, most of the patients prescribed clozapine opted for oral clozapine without ever receiving an injection $(n=20 / 39)$ or changed to oral clozapine after just one $(n=6 / 39)$ or two $(n=4 / 39)$ injections. ${ }^{2}$ The oral daily dose equivalence of one injection was $12.5 \mathrm{mg}$ and of two it was $25 \mathrm{mg}$. Among the 19 patients who received intramuscular clozapine, the median maximum daily dose was $75 \mathrm{mg}$ (range $6.25-200 \mathrm{mg}$ ), equivalent to $150 \mathrm{mg}$ of oral clozapine, suggesting that it may have been the assertive approach to non-concordance, rather than any antipsychotic effect of the intramuscular formulation, that facilitated the recommencement of oral medication (see also ${ }^{4}$ ). Nine (47\%) of their 19 patients needed physical restraint for the administration of the intramuscular clozapine. ${ }^{2}$ It is not clear whether any of the patients needed physical restraint for the venepuncture necessary for neutrophil monitoring.

\section{Safety and logistics}

Nevertheless, the use of intramuscular clozapine presents a number of safety and logistical considerations. Although previously a licensed product in several European countries, Sandoz (the original supplier) has ceased the production of intramuscular clozapine and it is currently unlicensed. This means that, as an unlicensed product in the UK and its country of origin, it falls within the highest-level risk category in the Medicines and Healthcare products Regulatory Agency (MHRA) hierarchy of medicines availability. ${ }^{5}$ In addition, from a medicines safety perspective, administration errors with intramuscular clozapine would be high risk, especially given the unfamiliarity with its use. The initial dose is $0.25 \mathrm{ml}$ and a dose exceeding $4 \mathrm{ml}$ would need more than one injection site. If the full $5 \mathrm{ml}$ vial (i.e. $125 \mathrm{mg}$ injection, equivalent to $250 \mathrm{mg}$ oral clozapine) were to be inadvertently administered to a clozapine-naive patient, the result could potentially be fatal.

Strict clinical governance procedures would therefore be necessary for the use of such a product in the form of endorsement by the multidisciplinary team, pharmacy, the medical director and a 
second opinion approved doctor (SOAD). The medication would then need to be ordered on a named patient basis. This would likely result in a considerable delay between the decision to prescribe intramuscular clozapine and its actual administration. In the Casetta et al study, ${ }^{2}$ the delay was at least 2 weeks and it could be even longer if the intramuscular clozapine supply chain were to be destabilised by the effects of Brexit and the COVID-19 pandemic. Strategies to mitigate the medicines safety risks would also need to be developed, but even if appropriately administered, the injection volume might still necessitate more than one injection.

\section{A cautious conclusion}

So far, studies reporting intramuscular and nasogastric ${ }^{4}$ clozapine use, including the retrospective study by Casetta et $\mathrm{al}^{2}{ }^{2}$ have had small sample sizes and need replication through larger, prospective studies with longer follow-up periods. However, until such data become available, intramuscular clozapine may be a suitable option in in-patient settings after appropriate staff training and rigorous clinical governance, for a small number of detained patients who can tolerate the delay to commencement of its use and who are not better served by alternative intramuscular antipsychotic injections - such as olanzapine or haloperidol, which are licensed (and hence quality assured), less expensive, have an established evidence base and are used with greater familiarity in clinical practice.

Shaun Bhattacherjee (D), BMedSci, BM BS, MMedSc, FRCPsych, Cumbria

Northumberland, Tyne and Wear NHS Foundation Trust, Whitehoven, UK;

Anthony Young BSC, MSC, IP(cert). MRPharmS, Cumbria, Northumberland, Tyne and

Wear NHS Foundation Trust, Newcastle upon Tyne; and Sunderland University School of

Pharmacy, UK; Niraj Ahuja, MB BS, MD, FRCPsych, PG Cert ClinRes, Cumbria,

Northumberland, Tyne and Wear NHS Foundation Trust, Newcastle upon Tyne; and

Department of Psychiatry, Newcastle University, UK; Steven Allen, MPharm (Hons),
IPresc, PGDipClinPharm, Cumbria, Northumberland, Tyne and Wear NHS Foundation Trust, Newcastle upon Tyne, UK

Correspondence: Dr S. Bhattacherjee. Email: shaun.bhattacherjee@cntw.nhs.uk

First received 12 Aug 2020, final revision 5 Feb 2021, accepted 16 Feb 2021

\section{Funding}

This research received no specific grant from any funding agency, commercial or not-for-profit sectors.

\section{Declaration of interest}

None.

\section{References}

1 Wyatt S, Aldridge S, Callaghan D, Dyke A, Moulin L. Exploring Mental Health Inpatient Capacity across Sustainability and Transformation Partnerships in England. Strategy Unit, Midlands and Lancashire NHS, 2019.

2 Casetta C, Oloyede E, Whiskey E, Taylor DM, Gaughran F, Shergill SS, et al. A retrospective study of intramuscular clozapine prescription for treatment initiation and maintenance in treatment-resistant psychosis. Br J Psychiatry 2020; 217: 506-13.

3 Tungaraza TE, Farooq S. Clozapine prescribing in the UK: views and experience of consultant psychiatrists. Ther Adv Psychopharmacol. 2015; 5: 88-96.

4 Till A, Selwood J, Silva E. The assertive approach to clozapine: nasogastric administration. BJPsych Bulletin 2019; 43: 21-6.

5 Medicines and Healthcare products Regulatory Agency. The Supply of Unlicensed Medical Products. Medicines and Healthcare products Regulatory Agency, 2014 (https://assets.publishing.service.gov.uk/government/uploads/ system/uploads/attachment_data/file/373505/The_supply_of_unlicensed_ medicinal_products specials_pdf). 